\title{
A PRELIMINARY DESIGN OF THE COLLINEAR DIELECTRIC WAKEFIELD ACCELERATOR
}

\author{
A. Zholents \#, W. Gai, S. Doran, R. Lindberg, J.G. Power, N. Strelnikov, Y. Sun, E. Trakhtenberg, I. \\ Vasserman, ANL, Argonne, IL 60439, USA \\ C. Jing, A. Kanareykin, Y. Li, Euclid Techlabs LLC, Solon, OH 44139, USA \\ Q. Gao, Tsinghua University, Beijing China \\ D.Y. Shchegolkov, E.I. Simakov, LANL, Los Alamos, NM 87545, USA
}

\begin{abstract}
A preliminary design of the multi-meter long collinear dielectric wakefield accelerator that achieves a highly efficient transfer of the drive bunch energy to the wakefields and to the witness bunch is considered. It is made from $\sim 0.5 \mathrm{~m}$ long accelerator modules containing a vacuum chamber with dielectric-lined walls, a quadrupole wiggler, an rf coupler, and BPM assembly. The single bunch breakup instability is a major limiting factor for accelerator efficiency, and the BNS damping is applied to obtain the stable multi-meter long propagation of a drive bunch. Numerical simulations using a 6D particle tracking computer code are performed and tolerances to various errors are defined.
\end{abstract}

\section{INTRODUCTION}

The concept of a soft x-ray Free-Electron Laser (FEL) facility based on a collinear dielectric wakefield accelerator (DWA) is proposed in Ref. [1]. One of the goals discussed there is a rapid acceleration of a low charge witness electron bunch to $2 \mathrm{GeV}$ in one accelerating stage using a single $400 \mathrm{MeV}$ high charge drive bunch. Using more than one accelerating stage implies having more than one drive bunch as well as implementation of a tight synchronization between witness and drive bunches, a task that we initially want to avoid. Under this condition, attaining a high energy for the witness bunch critically depends on the ability to efficiently extract most of the drive bunch energy (until it is almost completely stopped) and transfer a good fraction of this energy to the witness bunch. This paper is focused on a discussion of what it would take to do it.

One component of a planned strategy is preparing the drive bunch with a characteristic double triangle peak current distribution [2-4]. This will help in achieving two important results, i.e., increasing the ratio of the maximum accelerating wakefield behind the drive bunch to the decelerating field inside the drive bunch, and obtaining a nearly uniform deceleration and energy loss for all electrons inside the drive bunch except a small group of electrons at the head of the bunch. The preliminary experimental results for this effort are reported in Ref. [5].

It has been shown in [6,7] that a single bunch breakup instability (BBU) limits the distance of how long the drive bunch can propagate in the dielectric-lined waveguide before it begins losing the electrons. As a result, the amount of energy that the drive bunch can deposit to the wakefields behind it is also limited. In this paper we consider a practical design of a DWA (extended over 20 $\mathrm{m}$ and extracting $\sim 80 \%$ of the drive bunch energy) where BBU is contained by employing the BNS damping $[8,9]$. Implementation of the BNS damping entails using a strong focusing FODO channel whose purpose is to induce betatron oscillations of electrons with a short period commensurable to the small size of the dielectriclined waveguide.

This paper has two parts. In the first part we describe specific details of the design of the cylindrical dielectriclined waveguide and the FODO channel. In the second part we present the results of particle tracking through the DWA, demonstrating a self-deceleration of the drive bunch down to approximately $20 \%$ of its initial energy without the loss of the electrons.

\section{A DWA DESIGN}

Figure 1 shows a cross-section of the DWA. The grey part standing in the center is the vacuum chamber.

The strong-focusing quadrupole with the bore radius of $1.5 \mathrm{~mm}$ and the effective gradient of the magnetic field of $0.9 \mathrm{~T} / \mathrm{mm}$ surrounds the chamber, leaving a gap of 100 $\mu \mathrm{m}$ between the chamber wall and the magnetic poles. Four magnetic poles made from a soft magnetic material with a high saturation level of the magnetic flux density are supported by two non-magnetic strongbacks made from titanium. The strongbacks are connected at the top and at the bottom by two blocks made from invar. Two dashed trapezoids in the middle are the $\mathrm{NdFeB}$ permanent magnets. The ends of the magnets are recessed from the pole tips by $3 \mathrm{~mm}$ to avoid the area with a strong demagnetization field. Guided by the screws, the poles can slide along the magnets by $\pm 50 \mu \mathrm{m}$ and provide a fine adjustment of the quadrupole's magnetic center position in the horizontal $(x)$ and vertical $(y)$ directions in a range of $\pm 2.5 \mu \mathrm{m}$. The aim is to align magnetic centers of all quadrupoles inside the DWA module discussed below with an accuracy better than $0.75 \mu \mathrm{m}$ rms using pulsed wire magnetic measurements similar to the ones discussed in Ref. [10]. 


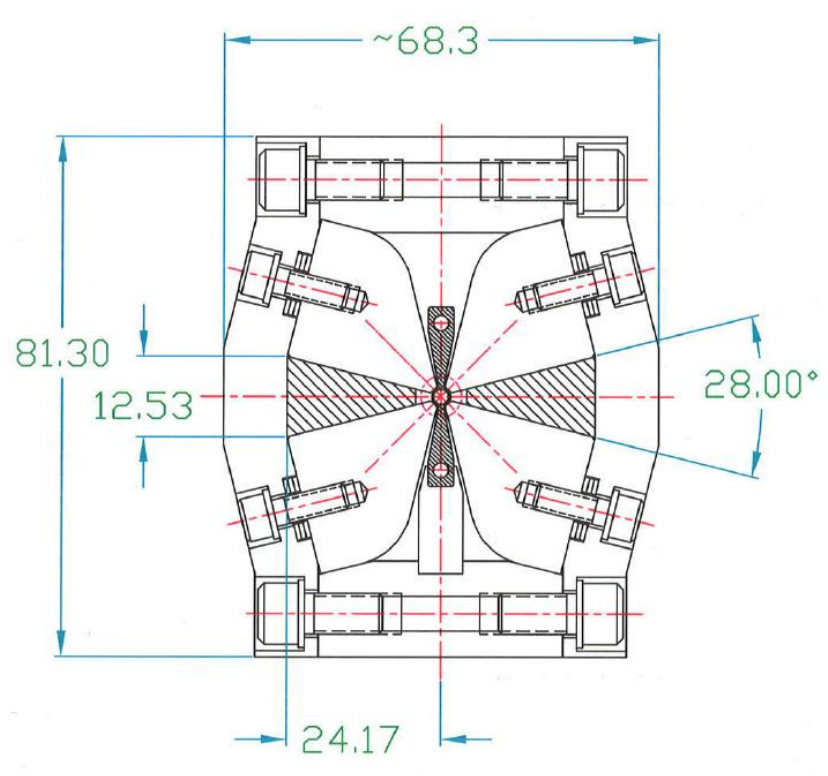

Figure 1. A schematic view of the DWA. All dimensions are given in $\mathrm{mm}$.

An assembly of ten $40 \mathrm{~mm}$ long quadrupoles, with alternating focusing and defocusing properties, is stacked into a $43 \mathrm{~cm}$ long quadrupole wiggler (Figure 2). A $3.5 \mathrm{~mm}$ drift space between the quadrupoles is occupied by four permanent magnets magnetized in the longitudinal direction. These magnets have the same contours as the magnetic poles and are used to eliminate a "cross-talk" of the neighbouring quadrupoles as shown in Figure 3.

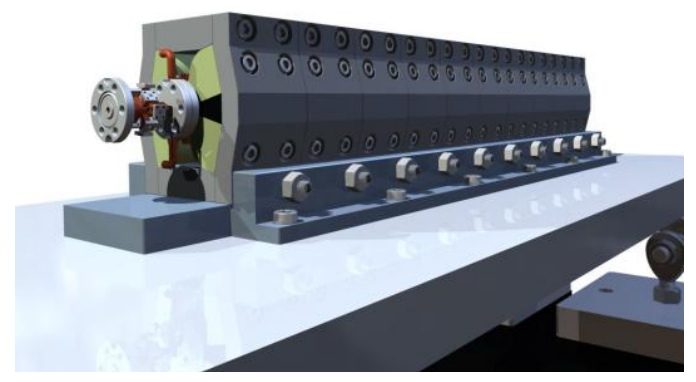

Figure 2. A quadrupole wiggler.

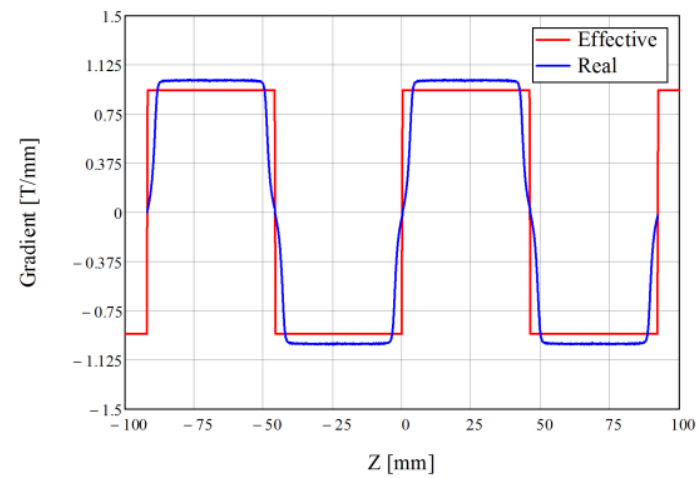

Figure 3 . The variation of the gradient of the magnetic field calculated on the axis of the quadrupole wiggler (blue curve) and the effective gradient (red curve). The field integrals under the blue and red curves are equal.

Once the ten quadrupoles have been aligned with respect to the center of a quadrupole wiggler, the entire quadrupole wiggler must be aligned to the neighbouring quadrupole wigglers. To do this, each quadrupole wiggler is mounted on a strongback and fixed using a series of heavy spring washers and set screws (similar to positioning the magnet poles). The strongback is moved using a system of $\mathrm{X}-\mathrm{Y}$ positioners secured to $\mathrm{Z}$ positioners in three locations. This results in quadrupole wigglers that can be aligned with an accuracy of $0.25 \mu \mathrm{m}$ in the $x-y$ directions and $1 \mu \mathrm{m}$ in the $z$ directions.

The vacuum chamber is shown in Figure 4. (It is rotated by $90^{\circ}$ for the purpose of presentation). It has three cylindrical holes and the plan is to make it with extrusion from a copper. The wall of a $2 \mathrm{~mm}$ diameter central pipe is covered by a $\sim 100 \mu \mathrm{m}$ thick dielectric layer (not shown in the drawing). The drive and witness bunches passing through this pipe at a $50 \mathrm{kHz}$ pulse repetition rate subject its wall to a heat load caused by the wakefields at a level of $\sim 10 \mathrm{~W}$ per every linear $\mathrm{cm}$ of the pipe (see [1]). Therefore, the vacuum chamber has on each side a $2.4 \mathrm{~mm}$ diameter pipe for water cooling that allows the highest vacuum chamber temperature to be maintained below $\sim 50^{\circ} \mathrm{C}$.

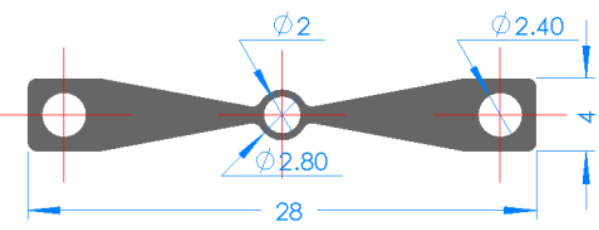

Figure 4. A vacuum chamber. All dimensions are given in $\mathrm{mm}$

Some of the challenges for a high repetition rate, high frequency DWA include heating of the vacuum chamber by the wakefields and a tight tolerance on a straightness of the beam trajectory. To address these issues, the vacuum chamber is equipped with the rf coupler and beam position monitor (BPM) assembly 
located in the $3.5 \mathrm{~cm}$ long break sections between quadrupole wigglers. Figure 5 shows this assembly. Here ports 1-4 are TM01 mode couplers designed to extract the residual RF energy out of the DWA structures with a better than $90 \%$ efficiency, reducing the heat load on the vacuum chamber (see Table 1). Meanwhile, the HEM11 mode, a dominant dipole mode excited by an off-axial beam in DWA structure, will naturally convert to the TE11 mode in the empty circular waveguide. Due to the pattern of surface current supporting the TE11 mode, the rf coupler and choke will not stop its propagation. Three TE11 mode couplers (ports 5, 6 and 7), which are 120 degrees apart, will couple out the dipole mode signals. Based on the signal strength of each of the three ports, the angular and radial position of the beam at the center of the upstream DWA structure can be fully restored, i.e. not at the location of the BPM itself, as in the case of the conventional cavity-type BPM. This design is very compact and uses only $3 \mathrm{~cm}$ (flange to flange) of space. The ports are also used for the vacuum pumping of each DWA module.

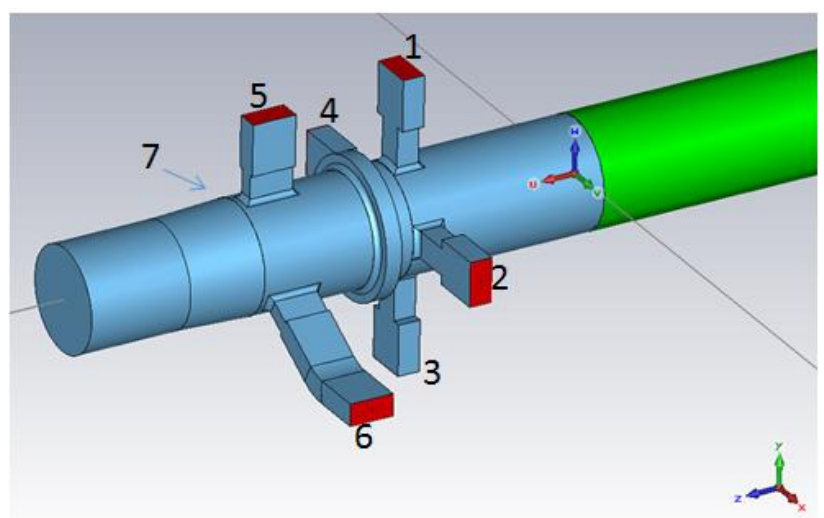

Figure 5. The 3D RF modeling for RF coupler and BPM assembly. Green is the dielectric layer and blue represents vacuum volume. The background is the perfect conductor.

Thus, the standard module of the DWA contains the quadrupole wiggler, the vacuum chamber, the rf coupler and BPM assembly, and the bellows (not shown in Figure 5). The DWA discussed in [1] will have $\sim 20$ such modules.

\section{PARTICLE TRACKING}

Particle tracking in a cylindrical dielectric-lined waveguide embedded into a quadrupole wiggler was performed in elegant [11] using the electron beam and waveguide parameters shown in Table 1 . Both the longitudinal and transverse wakefields were accounted for via Green functions calculated using the method described in Ref. [12]. The focusing strength of the quadrupole wiggler was optimized for better BBU suppression. The BNS damping was employed with the drive bunch having a negative energy chirp of $15 \%$ at the entrance of the DWA. Figure 6 shows the peak current of the beam at the beginning of the DWA, after $10 \mathrm{~m}$ of propagation and after $20 \mathrm{~m}$ of propagation, at which point the drive bunch has already lost almost $80 \%$ of its energy with no particle loss. Similar results were reported in Ref. [13] where quadrupoles of a fixed length of $4 \mathrm{~cm}$ and magnetic field gradient of $1.3 \mathrm{~T} / \mathrm{mm}$ were used. Here we used much weaker quadrupoles with $0.9 \mathrm{~T} / \mathrm{mm}$ magnetic field gradient and $3.5 \mathrm{~mm}$ spacing between the quadrupoles, but we varied the length of the quadrupoles along the DWA in order to match the decreasing energy of the drive bunch with the optimized focusing. The result is shown in Figure 7.

An addition of random quadrupoles' misalignments produced premature particle losses in simulations due to BBU when the rms misalignment error exceeded 0.75 $\mu \mathrm{m}$. The tolerance to the alignment of the quadrupole wiggler modules is a bit relaxed $<2 \mu \mathrm{m}$ rms.

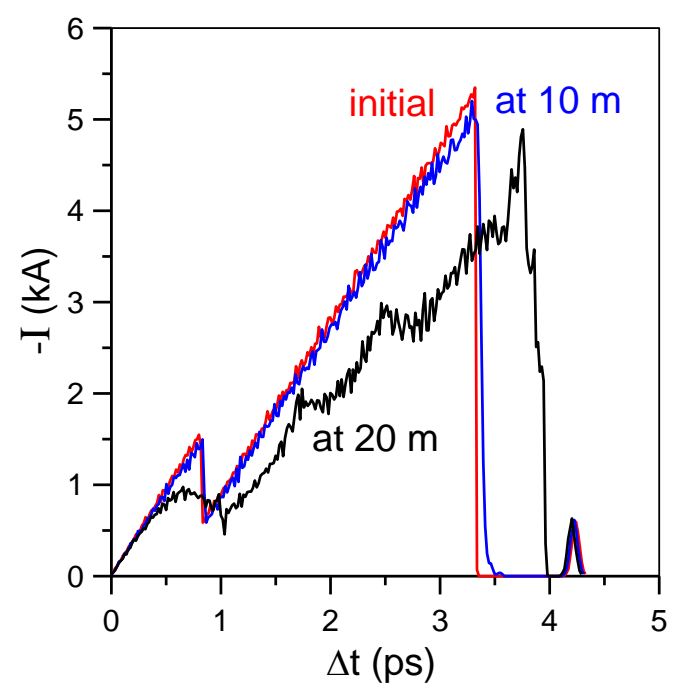

Figure 6. Current profile of the drive and witness bunches at different locations along the DWA. The drive bunch peak current distribution begins to degrade after approximately $10 \mathrm{~m}$ of propagation.

\section{TABLE 1. PARAMETERS USED FOR DWA SiMULATIONS}

\begin{tabular}{ll}
\hline \hline Parameter & \multicolumn{1}{c}{ Value } \\
\hline Main accel. mode frequency & $300 \mathrm{GHz}$ \\
Drive bunch charge/length & $8 \mathrm{nC} / 1 \mathrm{~mm}$ \\
Main bunch charge/rms length & $50 \mathrm{pC} / 10 \mu \mathrm{m}$ \\
Initial beam offset & $5 \mu \mathrm{m}$ \\
Circular dielectric tube ID/OD & $2 \mathrm{~mm} / 2.123 \mathrm{~mm}$ \\
Dielectric constant & 3.75 \\
Unloaded peak accel. gradient & $90 \mathrm{MV} / \mathrm{m}$ \\
Initial particle energy/chirp & $400 \mathrm{MeV} / 15 \%$ \\
\hline \hline
\end{tabular}




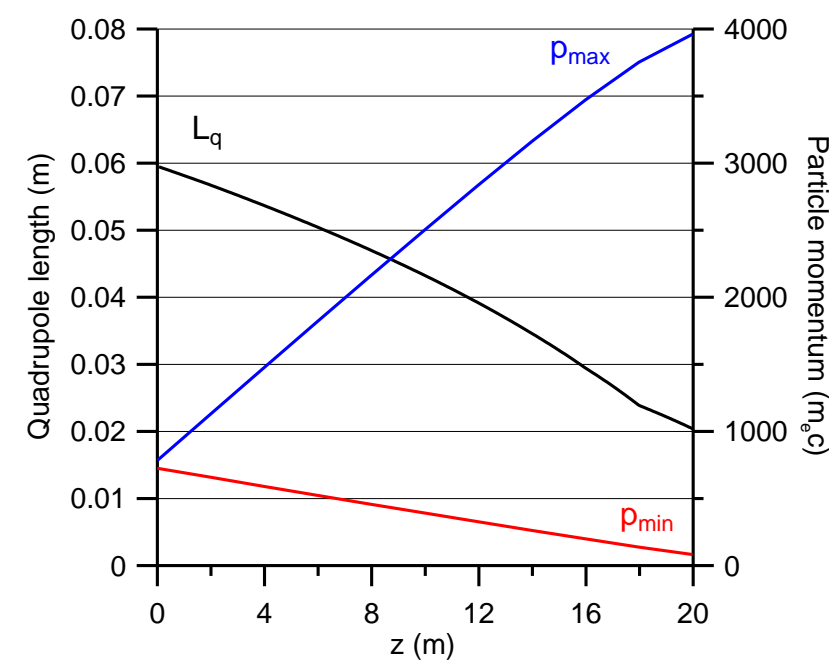

Figure 7. Optimized quadrupole length, $\mathrm{L}_{\mathrm{q}}$, and minimum and maximum particles' momentums in the drive and the witness bunches ploted versus the propagation distance.

\section{CONCLUSION}

A practical design of the DWA extending over 20 meters has been presented, and the possibility for a highly efficient transfer of the drive bunch energy to the wakefields and to the witness bunch has been demonstrated. Thanks to water-cooling of the vacuum chamber, the accelerator can operate at a high repetition rate up to $50 \mathrm{kHz}$. Several such accelerators can also share a single high repetition rate source of the drive and witness electron bunches and be assembled on a common supporting structure in one tunnel. This arrangement may be used for a multi-beamline, multi-user free-electron laser (FEL) facility where several FEL undulator lines can be driven by an equal number of high repetition rate single-stage collinear DWAs.

ACKNOWLEDGEMENT. This work was supported by the Office of Science of the U.S. Department of Energy under Contract No. DE-AC02-06CH11357 and by the U.S. Department of Energy through the Laboratory Directed Research and Development (LDRD) program at Los Alamos National Laboratory.

\section{REFERENCES}

[1] A. Zholents, W. Gai, R. Lindberg, J.G. Power, Y. Sun, C. Jing, et al., in: Proceedings of the 2014 FreeElectron Laser Conference, Basel, Switzerland, 2014.

[2] T. Katsouleas et al., "Beam loading in plasma accelerators," Particle Accelerators, vol. 22, pp.8199, 1987.

[3] B. Jiang, C. Jing, P. Schoessow, J. Power, and W. Gai, Phys. Rev. ST Accel. Beams, vol. 15, 011301, pp. 1-8, Jan. 2012.

[4] D. Yu. Shchegolkov and E. I. Simakov, Phys. Rev. ST Accel. Beams, vol. 17, 041301, pp. 1-10, Apr. 2014.

[5] J. Power, A. Zholents, K-J.Kim, M. Conde et al., in: Proceedings of the 2015 International Particle Acc. Conference, Dresden, Germany, 2014.
[6] C. Li et. al., "High Gradient Limits due to Single Bunch Beam Breakup in a Collinear Dielectric Wakefield Accelerator," Phys. Rev. ST Accel. Beams, to be published.

[7] D.Yu. Shchegolkov, E.I. Simakov, C. Jing, C. Li, A.A. Zholents, J.G. Power, Suppressing Parasitic Effects in a Long Dielectric Wakefield Accelerator, AAC2014 Conf. Proc. (to be published).

[8] V. Balakin et al., "VLEPP: Transverse Beam Dynamics," Proceedings of the $12^{\text {th }}$ International Conference on High Energy Accelerators, Fermi National Acceleratory Laboratory, Batavia, IL, 1983, p. 119.

[9] A. W. Chao, Physics of collective beam instabilities in high energy accelerators. New York: Wiley, 1993, ch. 3 .

[10] A. Alexandrov, A. Mikhailichenko, V. Parkhomchuk, A. Sery, V. Shiltsev, "Low aperture magnetic elements measurements," Preprint 91-70, Institute of Nuclear Physics, Novosibirsk, 1991.

[11] M. Borland, "elegant: a flexible SDDS-compliant code for accelerator simulation," in $6^{\text {th }}$ ICAP2000, Darmstadt, Germany; Advanced Photon Source LS287, pp. 1-11, Sept. 2000.

[12] K.-Y. Ng, "Wake fields in a dielectric-lined waveguide," Phys. Rev. D, vol. 42, no. 5, pp. 1819-1828, Sept. 1990.

[13] D.Y. Shchegolkov, E.I. Simakov, A.A. Zholents, IEEE Trans. Nucl. Sci., vol. 62 (6), pp. 1-8, Dec. 2015, 10.1109/TNS.2015.2482820. 